\title{
DIRECTORY OF IUGS BODIES AND AFFILIATED OR RELATED ORGANIZATIONS
}

COMMISSIONS

Commission for Marine Geology

Secretary: Dr. R.A. Scrutton,

Grant lnstitute of Geology

University of Edinburgh, West Majns Road

Edinburgh EH9 37 W, U.K.

Commission on Meteorites

Sect

Albuquerque, New Mexico 87106 , U.S.A.

Commission on Stratigraphy

Chairman: Prof. Anders Martinsson,

Department of Palaeobiology, Box 564

S-75| 22 Uppsala, Sweden

Secretary General: Dr. M.G. Bassett, Department of Gcology, National Muscum of Wales, Cardiff CFI 3NP, U.K.

Subcommission on Precambrian Stratigraphy

US Geological Survey, Box 25046, MS 912

Federal Center, Denver, Colorado 80225, U.S.A.

Working Group on the Precambrian-Cambriar

Boundary

Chairman: Dr. J.w. Cowic

University of Bristol, Department of Geology

Queen's Building, University Walk

Bristo! BSS ITR, U.K.

Subcommission on Cambrian Stratigraphy

Secretary: Dr. J.W. Cowie.

(See address above)

Working Group on the Cambrian-Ordovician Boundar

Secretary: Dr. D. Skevington,

Memorial University of Newfoundland

Department of Geology, St. John's, Nfld.

Canada AIC 5S7

Subcommission on Ordovician Stratigraphy

Department of Geology, Ohio State University

Columbus, Ohio 43210 , U.S.A.

Working Group on the Ordovician-Silurian

Boundary

Chairman: Dr. R.B. Richards,

Cambridge CB2 $3 \mathrm{EQ}$, U.K.

Subcommission on the Silurian System

Chairman: Prof. C.H. Holland,

Department of Geology, Trinity College

Dublin 2, Ireland

Subcommission on Devonian Stratigraphy

Chairman: Prof. W. Ziegler,

Department of Geosciences, Phillipps-University D-3550 Marburg/Lahn, F.R.G.

Working Group on the Devonian-Carboniferous Boundary

Convenor: Dr. Eva Paproth,

Geologisches Landesamt Nordrhein-Westfalen

De Greiff Str. 195. Postfach 1080

D-4150 Krefeld, F.R.G.

Subcommission on Carboniferous Stratigraphy

Secretary: Dr. C.F. Winkler Prins,

Rijksmuseum van Geologie en Mineralogic

Hooglandsekerkgracht 17

Leider. Netherlands

Subcommission on Gondwana Stratigraphy

Department of Geology, University of Sydney

Department of Geology,
Sydney, Australia 2600

Working Group on the Carboniferous-Permian

Boundary Group on the Carbon

Convenor: Dr. C.A. Ross,
Western Washington State College

Western Washington State

Department of Geology
Bellingham, Washington 98225 , U.5.A.

Subcommission on Permian Stratigraphy

Secretary: Prof. S.V. Meyen,

Geological Institute, Pyzhevsky per. 7

109017 Moscow, U.S.S.R.
Working Group on the Permian-Triassic

Boundary

Convenor: Prof. D.L. Stepanov,

Laboratory of Paleontology

Petroleum Geology Institute

Liteinyi Prospekt 39, Leningrad, D-104, USSR

Subcommission on Triassic Stratigraphy

Chairman: Dr. G. Richter Bernburg,

Bundesanstalt fur Geowissenschaften

Postfach 51 01 53, 3000 Hannover 51. F.R.G.

Subcommission on Jurassic Stratigraphy

Institut fur Palaeontologie der Universitat

Institut fur Palaeontologie der Universitat
Loewenichstrasse 28 , D-8520 Erlangen, F.R.G.

Working Group on the Jurassic-Cretaceous

Boundary

Co-Convenors: Dr. R. Casey,

Geological Institute, Exhibition Road,

South Kensington, London SW7 2DE, U.K.

Dr. D.C. Patrulius,

Institut de Cŕologie, Section Paléontologie

Sos. Kiscleff 55, Bucarest, Roumanie

Subcommission on Cretaccous Stratigraphy

Secretary: Dr. Finn Surlyk,

Geological Museum, University of Copenhagen

Oster Voldgade 5-7,DK-1350 Copenhagen, Denmark

Subcommission on Palacogene Stratigraphy

Chairman: Prof. Ch. Pomerol,

Laboratoire de Géologie des Bassins

F.75230 Paris Cedex 05. France

Working Group on the Palaeogene-Neogene

Boundary

Chairman: Dr. F. Steininger,

Palaontologisches Inst. der Universitat Wien

Universitatsstrasse ?/1I,

$A-1010$ Vienna, Austria

Subcommission on Neogene Stratigraphy

Instituto di Geologia, Museo Capellini

Via Zamboni 63-67, I-40127 Bologna, Italy

Rcgional Committee on Northern Neogene

Stratigraphy

Cr. Ph Cambridge.

258 Bluebell Road, Norwich NOR 66F. Norfolk, U.K.

Regional Committce on Mediterranean Neogene Stratigraphy

Chairman: Prof, J, Senes,

Geological Institute of the Slovak Academy

of Sciences, Dubravska resta

88625 Bratislava, C.S.S.R.

Regional Committec on Pacific Neogene Stratigraphy

Secretary: Dr. Yokichi Takayanagi,

Institute of Geology and Palaeontolog

Tohoku University Sendai, Japan

Working Group on the Neogene-Quaternary Boundary

Chairman: Prof. E. Aguirre,

Institute "Lucas Mallada"

J. Gutierrez Abascal 2, Madrid 6, Spain

Subcommission on Quaternary Stratigraphy

Seeres

Geologisches Institut der Universitat
Zulpicher Strasse 49, D 5000 Koln, F.R.G.

Regional Committee on Stratigraphic Correlation for the ESCAP Region

Chairman: Dr. Y. Shimazak.

Mineral Resources Section, Natura! Resources

Division, ESCAP. The United Nations Building

Rajadamnern Avenue, Bangkok 2, Thajland

Subcommission on Stratigraphic Classification

Chairman: Dr. Ános Salvador,

Exploration Department, Exxon Compan

P.O. Box 2180, Houston, Texas 77001, U.S.A.
Subcommission for the Stratigraphical Lexicon Chairman: Dr. C. Lorenz

Department de Geologie Structurale

4, Place Jussieu

F-75230 Paris Cedex 05, France

Subcommission on Geochronology

Chairman, Dr. W. Compston,

The Australian National University

Rosearch Sehool of Earth Sciences

Subcommission on Magnetic Polarity Time Scale

Interim Chairman: Dr. N. Opdyke

Lamont-Doherty Geological Observator

Commission on Systematics in Petrology

Chàrman: Prol. Dr. A. Streckelsen,

Mineralogisches-Petrographisches Institut

Sahlistrasse $6, \mathrm{CH}-30 / 2$ Bern, Switzeriand

Commission on Experimental Petrology at High

Pressures and Temperatures

Department of Geophysical Sciences

University of Chicago, 5734 South Ellis Avenue

Chicago, Illinois 60537, U.S.A.

Commission on Tectonics

Secretary: Prof. H.J. Zwart

Geologisch Instituut, Garenmarkt 18

Leiden, Netherlands

COMMITTEES

Committee on Geology Teaching

Department of Geology, Imperial College

Prince Consort Road, London, SW7 2BP, U.K.

Committee on Geological Documentation

Chairman: Dr. H. Glashoff

Bundesanstalt fur Geowissenschaften und Rohstoffe

Postfach 51 01 53, D-3000 Hannover 51, F.R.G.

Committee on Storage, Nutomatic Processing and Retrieval of Geological Data: (COGEODATA)

Bundesanstalt fur Geowissenschaften und Rohstoffe Postfach 51 01 53, D-3000 Hannover 5i, F.R.G.

Committce on the History of Geological Sciences (INHIGEO)

Chairman: Prof. R. Hooykaas,

Krullelaan 35, Zeist, Netherlands

Committee on Geoscience and Man

Convenor: Prot. Dr. G. Luttig,

Bundesanstalt fur Geowissenschaften und Rohstoffe

Postfach 51 01 53, D-3000 Hannover 51, F.R.G.

Advisory Board for Publication

Chairman: Dr. M.G. Bassett,

Department of Geology

National Museum of Wales, Cardiff CFI 3NP, U.K.

ASSOCIATIONS

Association Internationale pour l'Etude des Argiles (AIPEA)

Secretary: Prof. U. Schwertmann,

Institut fur Bodenkunde der Technischen

Hochschule Munchen

D-8050 Freising-Weihenstephan, F.R.G.

Association des Services Géologiques Africains (ASGA)

St. Michel

F-75272 Paris Cedex 06, France

Commission for the Geological Map of the World (CGMW)

Secretary: Miss F. Delany,

61, Boulevard de Montmorency

F-75016 Paris, France

European Association of Earth Science Editors SDITERRA

Secretary: Miss N.P. Morris,

30 Longdown Road, Lower Bourne

Farnham, Surrey, GU10 3IL, U.K. 